\title{
Modeling the Probability Distribution of Coordinates of Target, Detected by Two Sensors in Their Intersection Zone
}

\author{
Vladimir. D. Varbanov
}

\begin{abstract}
Sensors ability to accurately determine the coordinates of a target is often limited to one or two spatial coordinates, in example range at expense angle, as the accuracy is further degraded by noise of the signal. The problem can be mitigated by fusing the data of more than one sensor. The variance the fused coordinates can be evaluated theoretically or computationally through modeling. The paper focuses of the second approach.
\end{abstract}

Index Terms-Bivariate Distribution; Critical Infrastructure; Statistics; Sensors; Variance.

\section{INTRODUCTION}

The input signals of the sensors providing protection for critical infrastructure usually suffer interference and other distortions, which combined with limitations of the accuracy of the measurement result in difference between the measured coordinates of the intruder and the real ones [1][3]. The stochastic nature of these deviations defined as errors enable us to consider the coordinates random variables described by given distribution functions. The approaches for estimation of probability density function fall into two general categories - theoretical and empirical [4], [5], [7]. After the development of inexpensive computer hardware many of the empirical methods have strong focus on computing in the creation of new statistical methodology which leads to the development of the computational statistics. Its methods are concerned with estimating distributions via simulations.

\section{PURPOSE}

The focus at the paper is set on proposition and implementation of modeling the joint probability density function of the spatial coordinates, derived from the output data of several independent sensor devices, which monitor overlaying security zones. Various types of sensors are used as metric information sources regarding the coordinates of an intruder. The data obtained from them is described by different probability distributions, depending on the sensor type. Compiling that information could decrease the uncertainty of the estimated coordinates of the intruder but leads to the formation of complex joint probability distributions, whose attributes are challenging to be assessed. In order to resolve this problem, the paper propose

Published on April 29, 2018.

V. D. Varbanov is with the Institute of Metal Science equipment, and technologies with Center for Hydro- and Aerodynamics "Acad. A. Balevski" - Bulgarian Academy of Sciences, Department Technologies and systems for protection, Sofia 1574, Bulgaria (e-mail: vdvarbanov@abv.bg). implementation of computational statistic, which allow us to use statistics based on simulated data to gain understanding about desired probability distributions characteristics. Simulations have been conducted in programing environment MATLAB.

\section{STATEMENT}

Widely used technique among the empirical methods constitutes numerical modeling of the outcome of the target coordinates acquired by every sensor device separately, followed by processing the unified data obtained by several sensors incorporating computational statistics. We can simplify the procedure stated above by making the assumption that the presence of intruder has been sensed by two devices operating in overlapping zones [3], [4]. Let's presume that these sensors are intrusion detection sensor, which we will refer as barrier sensor (sensors which detects an intruder crossing a particular boundary defined by a line laying between the transmitter and receiver) and isotropic sensor (sensors with isotropic radiation pattern which are able to measure the distance to the target based on the magnitude of the received signal, but cannot evaluate its angular coordinates). In order to conduct the simulations, we should have a familiarity with probability density functions describing the spatial distribution of target's coordinates, associated with each sensor [3], [4]. The benefits in implementation of this approach are that the specific type of sensor, as well as the physical domain of the signals that are being processed are insignificant. Therefore, it can be applied for barrier sensors of different type, in example infrared, laser, optic, electro-mechanical and others. The same is viable for the isotropic sensors, which can be seismic, acoustic or thermal omnidirectional.

In the following section we show two of the most versatile scenarios - the cases where an intruder has been detected in the overlaying operating zone by barrier and isotropic sensor and a case where two isotropic sensors are used. Recall that the spatial point generated pattern is distributed according to some probability function we need to define the functions describing the associated sensor types [1], [6], [7].

The output metric information retrieved by barrier sensor provide data about the presence of a target with subordinate complex coordinates $l=x+i y$ positioned with equal probability

$P(l)=\int_{a}^{b} F(l) d l=\frac{l-a}{b-a}$

upon the line defined by the coordinates of the receiver 
$a=x_{r}+i y_{r}$ and the transmitter $b=x_{t}+i y_{t}$ and the probability density function:

$f(l)=\frac{1}{b-a}=$ const

In the case of isotropic sensor, the distance to the target can be measured based on the magnitude of the received signal, but the same does not apply for its angular coordinates. Therefore, we can make the assumption that probability density function of the distance to the target is characterized by normal distribution, while of the angular coordinates by uniform distribution. Consecutively, the probability density function describing the possible location of an intruder can be denoted by the following equation:

$$
f(r)=f\left(x_{c}, y_{c}\right)=\frac{1}{\sqrt{2 \pi \sigma}} e^{-\frac{1}{2}\left[\frac{\left(\sqrt{\left(x_{c}-x_{0}\right)^{2}+\left(y_{c}-y_{0}\right)^{2}}\right)^{2}-R^{2}}{\sigma}\right]}
$$

Where $r=\sqrt{\left(x_{c}-x_{o}\right)^{2}+\left(y_{c}-y_{0}\right)^{2}}$ is the range between the measured coordinates of the target $x_{c}, y_{c}$ and the location of the sensor $x_{0}, y_{0}$, while $R$ is the mean value of that distance[2], [4].

After spatial distribution of target's coordinates, associated with each type of the given sensors, have been defined by (2) and (3) we can turn our attention to estimating the complex joint probability density functions $f(\zeta)$ and $f(\xi)$, which describe the stated scenarios - the intruder has been detected by barrier and isotropic sensor or by two isotropic sensors[3], [4], [5].

The steps required for the implementation of an approach based on computational statistics, have been outlined below:

\section{A. Modeling the base probability distributions comprising the joint probability distribution}

A representative sample of size $\mathrm{n}$ is generated, consisting of the realization of the random variables $l$ and $r$, distributed according to the desired probability density functions $f(\zeta)$ and $f(\xi)$ on the associated axes. The observation of the modeled spatial data points leads to two interesting revelations - the presence of more than one global maximum and difference between the calculated expected values:

$m_{\xi x}=29.74 m_{\xi y}=27.53 m_{\zeta x}=27.50 m_{\zeta y}=23.01$

and the coordinates where the probability density functions of the sensors $f(l)$ and $f(r)$ cross each other - $(35 ; 40)$, which is the most probable location of the intruder. Therefore, at this stage of processing of the statistical information, the mean value should not be considered solution of the location problem.

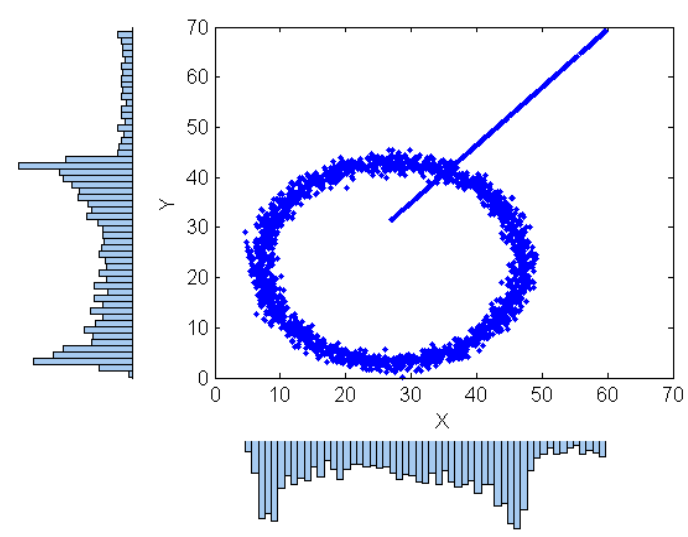

(a)

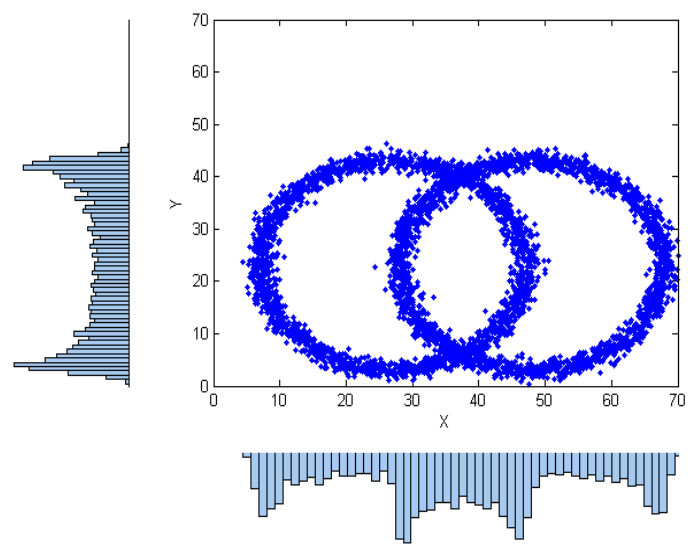

(b)

Fig. 1. 1a) Simulation of observations obtained by isotropic and barrier sensors characterized by density function $f(\xi), 1$ b) Simulation of observations obtained by two isotropic and sensors characterized by density function $f(\zeta)$.

\section{B. Construction of bivariate histogram}

For interpretation of observations it is usually necessary a histogram to be created. Histograms are a good way to summarize a data set in order to understand general characteristics of the distribution such as shape, spread or location [8], [9]. In this section we will focus our attention to the bivariate normalized density histogram corresponding to the discretized probability density function $\hat{f}(\xi)$ of continuous variable $\xi$, analogically $\hat{f}(\zeta)$ and $\zeta$, given by equation:

$\hat{f}(\xi)=\frac{v_{i j}}{n h_{i} h_{j}} \quad \xi \in B_{i j}$

Where $B_{i j}$ denotes the associated bin, while $v_{i j}$ represents the number of observations falling into the bivariate bin $B_{i j}$ and $h_{i}, h_{j}$ govern its size. 
(a)

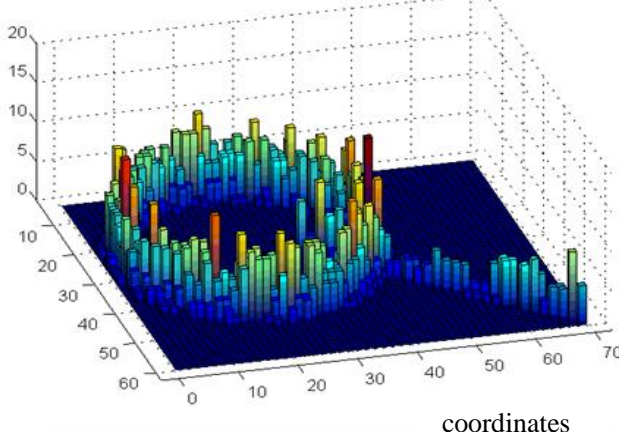

(b)

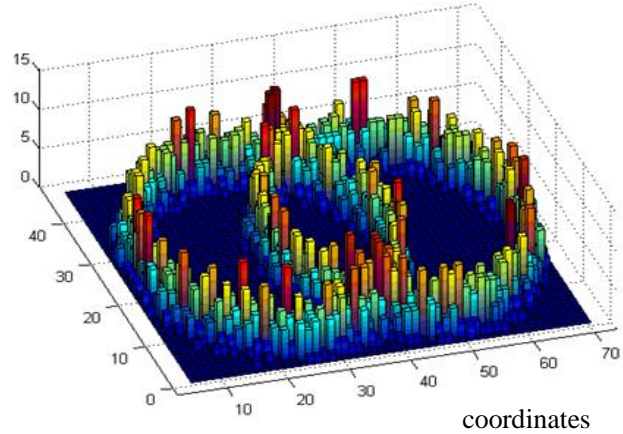

Fig. 2. 2a) Bivariate histogram with discretized probability density function $\hat{f}(\xi), 2$ b) Bivariate histogram with discretized probability density function $\hat{f}(\zeta)$

C. Find the cell $B_{i j}$ of the histogram, that yields the highest value $v_{i j}\left(B_{i j}\right)=$ max and explore the characteristics of density function in an area defined around it.

Because of the ring shape of the density functions $\hat{f}(\xi)$ and $\hat{f}(\zeta)$, the expected value does lay around the center of the gauss ring and not on the cross point of the two distribution functions of the two sensors. Consequently, it would be sensible to define an area with radius equal to the standard deviation $D=\sqrt{\sigma^{2}}$, centered at the global maximum, satisfying the condition $v_{i j}\left(B_{i j}\right)=\max$ and explore its numerical values of the variance and the mean.
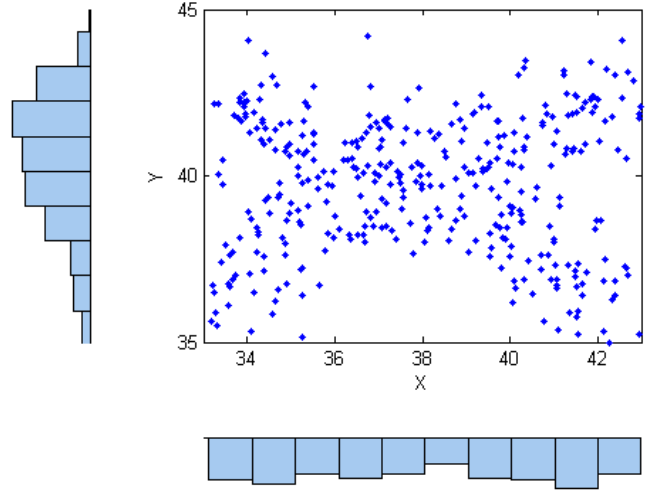

(a)
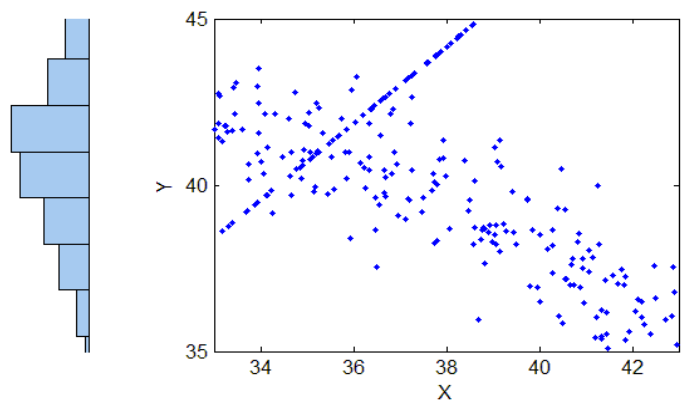

(b)

Fig.3. 3a) Distribution of the coordinates of the target around the most probable point of the density function $\hat{f}(\xi), 3 \mathrm{~b}$ ) Distribution of the coordinates of the target around the most probable point of the density function $\hat{f}(\zeta)$

A. Determination of the numerical values of the mean and variance of the data set by equations:

$\bar{x}=\frac{1}{n}\left(\bar{x}_{1}+\bar{x}_{2} \cdots+\bar{x}_{2}\right)$

$\sigma^{2}=\frac{1}{n-1}\left(x_{i}-\bar{x}\right)^{2}$

Fig. 3 shows that relationship exist between the angular slices of distribution function and the values, which the variance takes on. Rotating the coordinates of the models enable us to show the apparent dependence of the variance from the angle values.

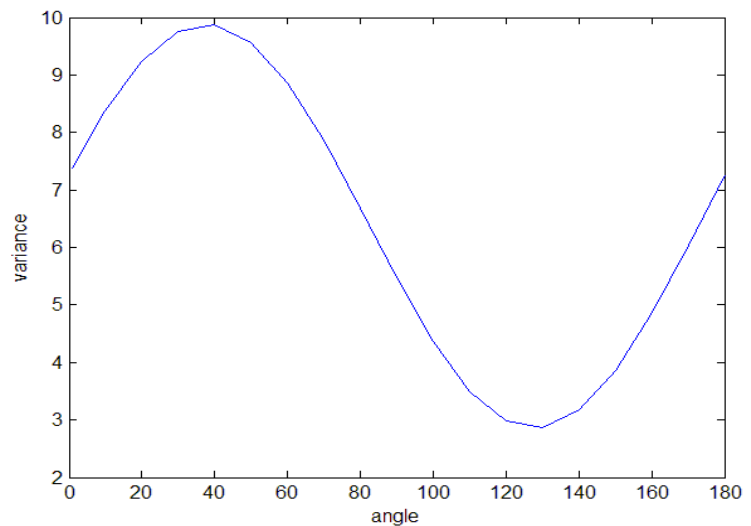

(a) 


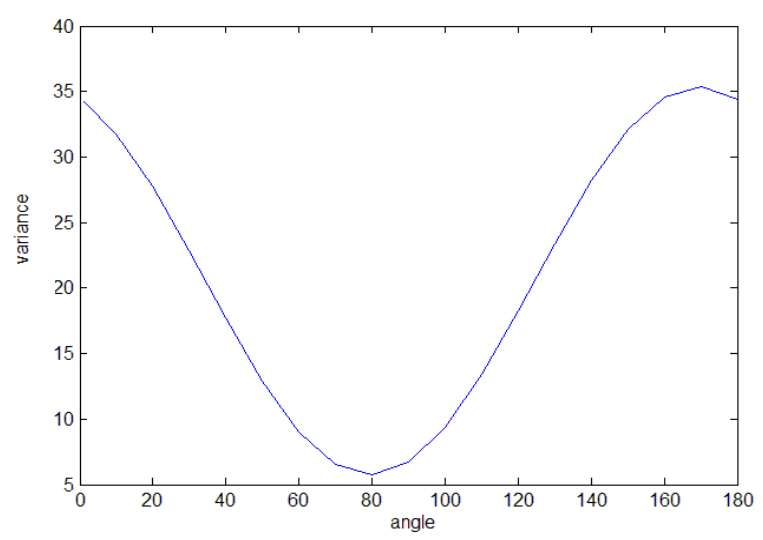

(b)

Fig. 4. 4a ) Plot of variance against angle in degrees for distribution $\hat{f}(\xi)$, 4b) Plot of variance against for distribution $\hat{f}(\zeta)$

\section{CONCLUSION}

Looking at (1) and (2) it becomes apparent that determination of the eventual coordinates of an intruder in critical infrastructure zone with only one sensor will be inaccurate, due to the uniform component of the equation describing probability density function of observed types of sensors. Therefore, the successful solution of the location problem require surveillance in the secured sector to be conducted by two sensors with overlapping operating zones. In this case it is possible to construct joint probability function which can minimize the dispersive influence of the uniform component of the linear and angular distribution of the detected target.

\section{REFERENCES}

[1] N. L Gueourgiev, "Criteria for the Quality of Systems Used in Protect ion of Critical Infrastructure", NDT days, pp. 100-102, ISSN:1310 3946, Bulgaria, 2015.

[2] N. L Gueourgiev, "Time of Existence of Alarm Event for a Class of S ensors", NDT days, pp. 103-105, ISSN:1310-3946, Bulgaria, 2015.

[3] R. Andrew, "Approximation of Density Functions by Sequence of Ex ponential Families", The Annals of Statistics Vol. 19, No 3(Sep 1991) pp. 1347-1369.

[4] N. L Gueourgiev, "Generalized Quality Parameters of Sensors Used in Security Systems", NDT days, pp. 118-120, ISSN:1310-3946, Bulgaria, 2015.

[5] Y. Efroimovich, "Estimation of Square Integrable Probability Density of Random Variable", Problems Inform Transmission 18 pp. 175-189.

[6] J. Hough, "Determinantial Processes and independence, Probability S urveys 3", pp. 206-229, arXiv:math.PR/0503110.

[7] N. L Gueourgiev, "Calculation of the apostheory probabilities for the presence of objects according to sensor data", Journal of Engineering Sciences, vol. 3, pp. 16-25, 2016.

[8] N. L Gueourgiev, "Probability characteristics of false alarm signals of a class of sensors", Journal of the Technical University - Sofia, Plovdiv branch, Bulgaria, "Fundamental Sciences and Applications", vol. 2, pp. 106-109,2015

[9] N. L Gueourgiev, "Generalized description of the state of sensors used in security systems", NDT days 2015, pp. 118-120, ISSN:13103946, Bulgaria, 2015.

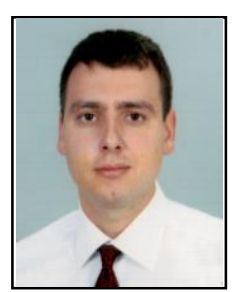

Vladimir D. Varbanov

City: Veliko Tarnovo, country: Bulgaria, date of birth: 21.10.1985. Bachelor degree of Engineering Physics at Sofia University "St. Kliment Ohrdiski", Department of Radio Physics and Electronics, Sofia, Bulgaria - 2009. Master degree of Engineering Physics at Sofia University "St. Kliment Ohrdiski", Department of Radio Physics and Electronics, Sofia, Bulgaria - 2011. PhD at Institute of Metal Science equipment, and technologies with Center for Hydroand Aerodynamics "Acad. A. Balevski" - Bulgarian Academy of Science, Sofia, Bulgaria - 2016.

After university graduation he continues his academic career at Institute of Metal Science equipment, and technologies with Center for Hydro- and Aerodynamics "Acad. A. Balevski" at the Bulgarian Academy of Science on technologist position. The job consists development and implementation of data fusion algorithms for use in multisensory systems and devices for security of critical infrastructure. Successfully defends doctor thesis at the same field in 2016. From 2016 work as assistant professor in domain of computer vision and machine learning. 